\title{
A Density Functional Study of Zinc Oxide Elastic Properties Under High Pressure
}

\author{
Bin-Bin Wang and Ya-Pu Zhao
}

\begin{abstract}
Zinc Oxide ( $\mathrm{ZnO}$ ) material has been widely used in piezoelectric devices, electro-optic devices, and biomedical sensors, etc. Among three crystal phases, $\mathrm{ZnO}$ with wurtzite structure (B4) is most common; thus it usually exists in natural $\mathrm{ZnO}$ materials. $\mathrm{ZnO}$ with zinc blend structure (B3) is another common crystal phase, which is metastable in nature. As observed by researchers, $\mathrm{ZnO}$ materials synthesized by chemical vapor deposition (CVD), which is one of the most popular methods to synthesize nanomaterials, have both B3 and B4 structures. In this paper, we discussed the elasticity and crystal structure of $\mathrm{ZnO}$ with the two structures under high pressure from 0 to $10 \mathrm{GPa}$. The CAmbridge Serial Total Energy Package (CASTEP) based on density functional theory (DFT) was used to perform the calculations. Both local density approximation (LDA) and generalized gradient approximation (GGA) were employed for comparison. We found that all the lattice constants decreased with the increasing pressure, and the relationship was linear, while the bulk moduli increased with the increasing environment pressure. However, the elastic constants (including $\mathrm{C}_{11}, \mathrm{C}_{12}$ and $\mathrm{C}_{44}$ for $\mathrm{B} 3 \mathrm{C}_{11}, \mathrm{C}_{12}, \mathrm{C}_{13}, \mathrm{C}_{33}$ and $\mathrm{C}_{44}$ for $\left.\mathrm{B} 4\right)$ showed a more sophisticated trend, which could be attributed to the particular symmetry of the crystal structure. The elastic constants denoting the elasticity of longitudinal directions would become larger with the increasing pressure, while the others show irregular trends. The results in this paper will be helpful in widening the application of devices based on $\mathrm{ZnO}$ nanomaterials.
\end{abstract}

Keywords $\mathrm{ZnO}$ - Elastic properties - Density functional theory (DFT) • Multi-scale simulation

B.-B. Wang • Y.-P. Zhao ( $\square)$

State Key Laboratory of Nonlinear Mechanics, Institute of Mechanics,

Chinese Academy of Science, Beijing 100190, China

e-mail: yzhao@imech.ac.cn 


$\begin{array}{ll}\text { Abbreviations } \\ \text { CAPZ } & \text { Ceperley-Alder -Perdew-Zunger } \\ \text { CASTEP } & \text { CAmbridge Serial Total Energy Package } \\ \text { CVD } & \text { Chemical Vapor Deposition } \\ \text { DFT } & \text { Density Functional Theory } \\ \text { FEM } & \text { Finite Element Method } \\ \text { GGA } & \text { Generalized Gradient Approximation } \\ \text { HF } & \text { Hartree-Fock } \\ \text { LDA } & \text { Local Density Approximation } \\ \text { MD } & \text { Molecular Dynamics } \\ \text { MM } & \text { Molecular Mechanics } \\ \text { QM } & \text { Quantum Mechanism } \\ \text { TBMD } & \text { Tight Binding Molecular Dynamics } \\ \text { ZnO } & \text { Zinc Oxide }\end{array}$

\section{Introduction}

Zinc Oxide $(\mathrm{ZnO})$, as the third generation semiconductor materials, has attracted much attention in the last decade. $\mathrm{ZnO}$ is a wide-band-gap semiconductor with a wide range of technological applications including electronic and electro-optic devices, catalysis, chemical sensors, and so on. Especially, $\mathrm{ZnO}$ film has been used as n-type conducting windows in thin film solar cells based on amorphous silicon. In recent years, many efforts have been made on the synthesis and applications of nanosized $\mathrm{ZnO}$ materials [1-4]. For example, we have investigated the junction structure of $\mathrm{ZnO}$ tetrapods, which would be helpful to understand the growth mechanism and applications of $\mathrm{ZnO}$ tetrapods [5]. ZnO occurs naturally as a mineral and its high pressure phase may be geologically important as a component of the lower mantle. $\mathrm{ZnO}$ usually crystallizes in three forms: hexagonal wurtzite (B4), cubic zincblende (B3) and the rarely observed cubic rocksalt (B1). The wurtzite structure is most stable under atmospheric condition and thus most common. The zinc blende form can be stabilized by growing $\mathrm{ZnO}$ on substrates with cubic lattice structure. In both cases, the zinc and oxide centers are tetrahedral. Besides, the rocksalt structure is only observed at relatively high pressures about $10 \mathrm{GPa}$.

The chemical vapor deposition (CVD) method is one of the simplest approaches, which has been widely used to produce $\mathrm{ZnO}$ nanostructures. Many studies reveal that the nanosized $\mathrm{ZnO}$ materials grown by CVD are wurtzite structured (B4), while the zinc blend (B3) structure exists relatively small in quantities [4, 6]. Furthermore, the elastic properties under high pressure should be studied extensively in order to expand the application of $\mathrm{ZnO}$ materials. The $\mathrm{B} 4$ structure is hexagonal, and there exists five independent elastic constants: $\mathrm{C}_{11}, \mathrm{C}_{33}, \mathrm{C}_{12}, \mathrm{C}_{13}$ and $\mathrm{C}_{44}$. Elastic constants $\mathrm{C}_{11}$ and $\mathrm{C}_{33}$ correspond to longitudinal modes along the [1000] and 


\section{Framework for Simulation}

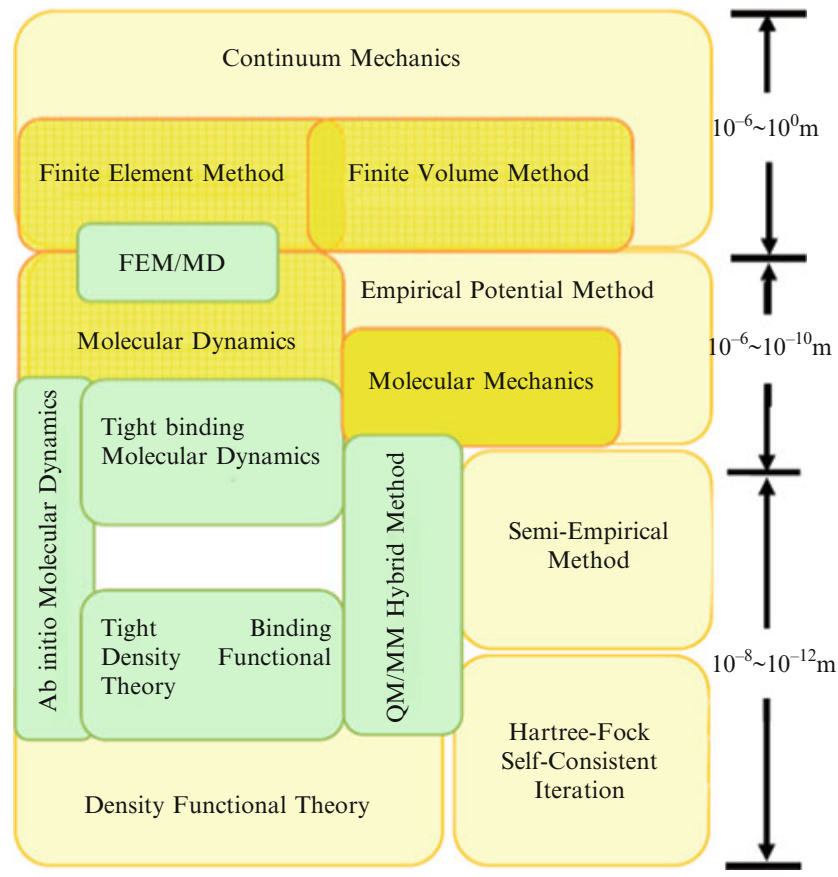

Fig. 1 Multi-scale simulation framework in the authors' group

[0001] directions, respectively. Elastic constants $\mathrm{C}_{44}$ and $\mathrm{C}_{66}=\left(\mathrm{C}_{11}-\mathrm{C}_{12}\right) / 2$, owing to symmetry, can be determined from the sound velocity of transverse modes propagating along the [0001] and [1000] directions, respectively. The remaining constant $\mathrm{C}_{13}$ is present in combination with four other moduli in the velocity of modes propagating in less-symmetrical directions, such as [0011]. The B3 structure has higher symmetry than $\mathrm{B}_{4}$; thus it has only three elastic constants denoted $\mathrm{C}_{11}$, $\mathrm{C}_{12}$ and $\mathrm{C}_{44}$.

The elastic properties of $\mathrm{ZnO}$ single crystals have been investigated by many researchers by both experimental and computational methods [7-12]. In this paper, we present the elastic constants and crystal constants of $\mathrm{ZnO}$ with $\mathrm{B} 3$ and $\mathrm{B} 4$ structures obtained by density functional theory (DFT), using both GGA and LDA approximations. Young's modulus and Poisson's ratio were also studied. Corresponding results would be helpful to widen the applications of $\mathrm{ZnO}$ materials in devices based on nanomaterials. Furthermore, the calculations were carried out on the simulation platform illustrated in Fig. 1, which had been constructed for multi-scale simulations, including DFT, quantum mechanics/molecular mechanics (QM/MM), tight binding molecular dynamics (TBMD), molecular dynamics (MD), and finite element method (FEM), etc. Results on multi-scale physical mechanics have attracted much attention in the corresponding field [13-15]. 


\section{Model}

The calculations were performed using the program CASTEP (CAmbridge Serial Total Energy Package), which uses DFT to provide a good atomic level description of all manner of materials and molecules. In our calculations, both LDA and GGA methods have been applied. The B3 and B4 structures are investigated in this paper, while B2 was not included because of its rareness. The crystal structures of both B3 and B4 are shown in Fig. 2.

CASTEP employs the DFT plane-wave pseudo potential method, which can perform first-principles quantum mechanics calculations that explore the properties of crystals and surfaces in materials such as semiconductors, ceramics, metals,

a
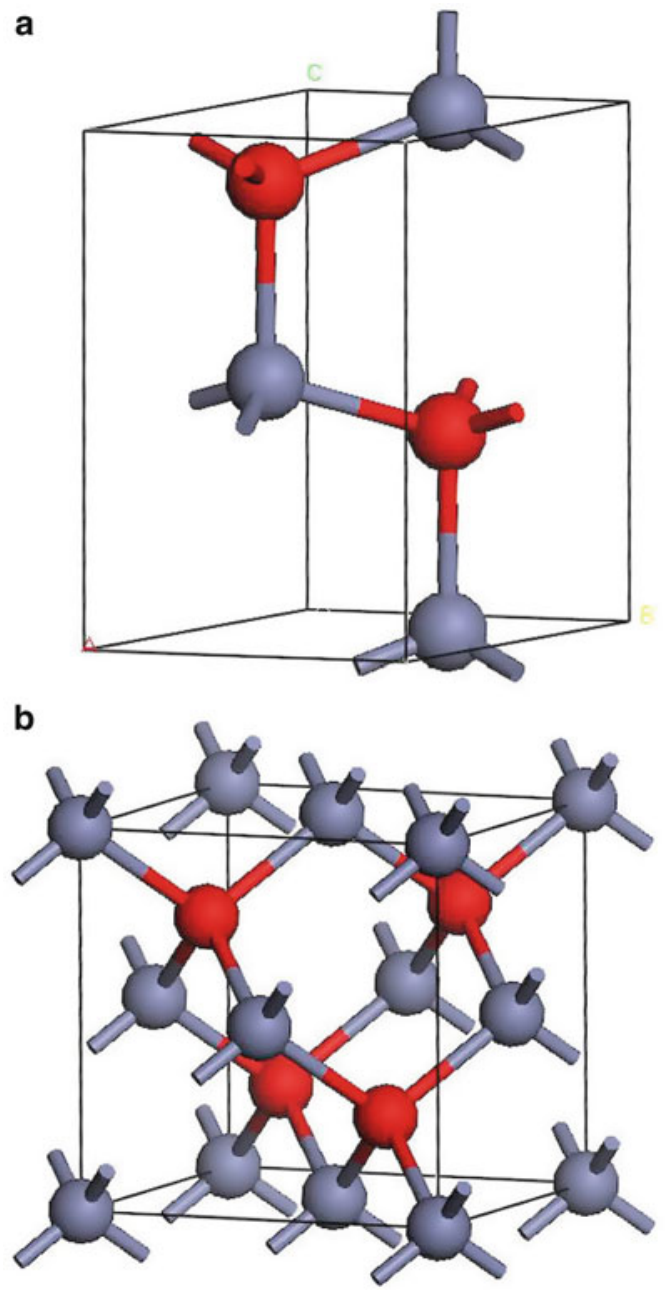

Fig. 2 Crystal structures of wurtzite and zinc blende $\mathrm{ZnO}$ : (a) wurtzite; (b) zinc blende 
minerals, and zeolites. For LDA and GGA methods, the Ceperley-Alder-PerdewZunger local-density approximation (CAPZ) $[16,17]$ and Perdew-Wang generalized gradient approximation (PW91) [18] were employed to calculate $\mathrm{ZnO}$ 's crystal properties. The environment pressure was applied as static hydrostatic pressure, and the values are $0,2,4,6,8,10 \mathrm{GPa}$.

\section{Results and Discussions}

The crystal structures for the $\mathrm{B} 3$ and $\mathrm{B} 4$ phases of $\mathrm{ZnO}$ are presented in Fig. 2. The wurtzite $\mathrm{ZnO}$ belongs to the $\mathrm{C}_{6 \mathrm{v} 4}\left(\mathrm{P}_{63 \mathrm{mc}}\right)$ space group. The primitive cell includes two formula units, with all atoms occupying $2 \mathrm{~b}$ sites of symmetry $\mathrm{C}_{3 \mathrm{v}}$. The structure is described by two lattice constants $a$ and $c$, and the internal parameter $u$, whereas the zinc blend structure is described by only one lattice constant $a$. Experimental results for lattice constant of B3 under atmospheic pressure is $a=4.58 \AA$, while $a=3.25 \AA$ and $c=5.2 \AA$ for B4.

Figure 3 shows the crystal constant of $\mathrm{B} 3 \mathrm{ZnO}$ decreases with increasing pressure, and the relationship is linear. Results with GGA are larger than the ones with LDA. Compared with experimental data, the actual value is between the results

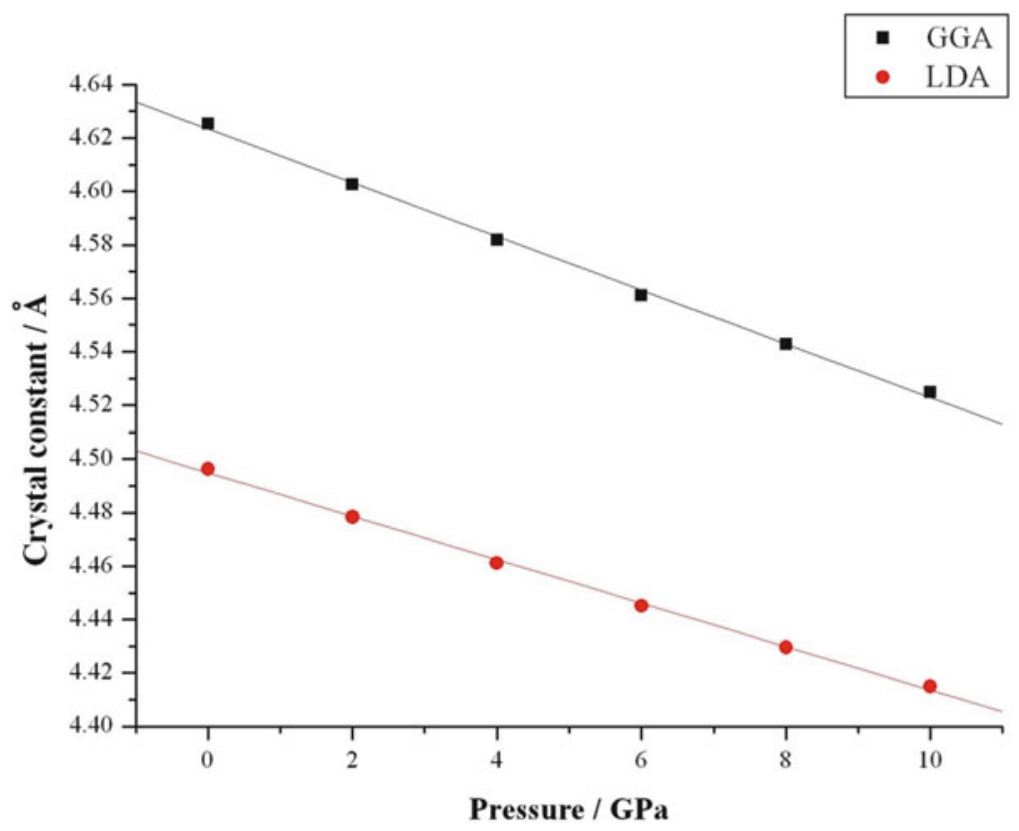

Fig. 3 Relationship between crystal constant and pressure for the $\mathrm{B}_{3}$ structure 


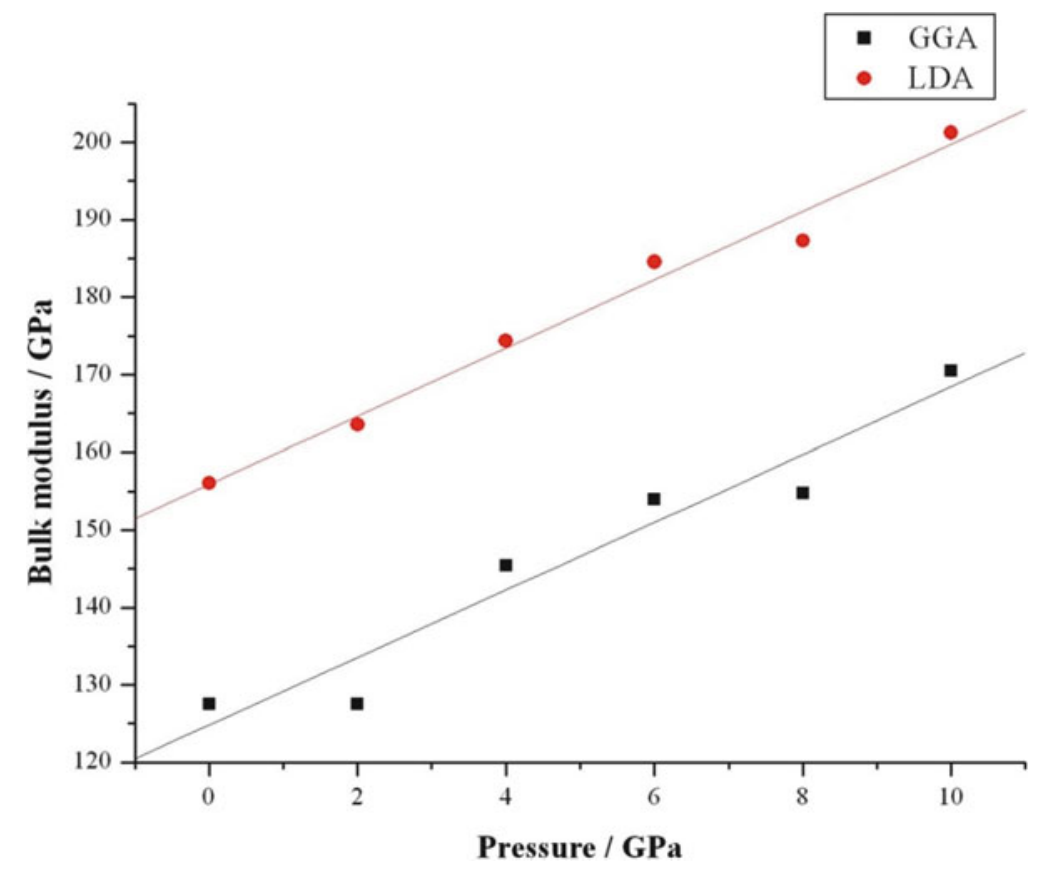

Fig. 4 Relationship between bulk modulus and pressure for the $\mathrm{B}_{3}$ structure

obtained using GGA and LDA. The bulk modulus of B3 $\mathrm{ZnO}$ presented in Fig. 4 shows a similar situation; however the trend is opposite: bulk modulus increases with increasing pressure.

Different crystal structures have different numbers of elastic constants and higher symmetry would reduce the numbers. For $\mathrm{ZnO}$ crystals, B3 belongs to the cubic crystal system, while $\mathrm{B} 4$ belongs to the hexagonal crystal system. Thus $\mathrm{B} 3$ has three independent elastic constants $\left(\mathrm{C}_{11}, \mathrm{C}_{12}\right.$ and $\mathrm{C}_{44}, \mathrm{C}_{11}=\mathrm{C}_{22}=\mathrm{C}_{33} ; \mathrm{C}_{12}=\mathrm{C}_{23}=\mathrm{C}_{31}$; $\left.\mathrm{C}_{44}=\mathrm{C}_{55}=\mathrm{C}_{66}\right)$ and $\mathrm{B} 4$ has five independent elastic constants $\left(\mathrm{C}_{11}, \mathrm{C}_{12}, \mathrm{C}_{13}, \mathrm{C}_{33}\right.$ and $\mathrm{C}_{44}, \mathrm{C}_{11}=\mathrm{C}_{22}, \mathrm{C}_{44}=\mathrm{C}_{55}, \mathrm{C}_{66}=\left(\mathrm{C}_{11}-\mathrm{C}_{12}\right) / 2$, and $\left.\mathrm{C}_{13}=\mathrm{C}_{23}\right)$. The results for $\mathrm{B} 3$ are shown in Fig. 5 and the tendency with high pressure is not consistent for elastic constant: $\mathrm{C}_{11}$ and $\mathrm{C}_{12}$ increase with increasing pressure, which reflects that the compact arrangement of atoms lead to large repulsion among atoms. The relationships with pressure are linear: $\partial \mathrm{C}_{11} / \partial \mathrm{P}=3.51(\mathrm{LDA}), \partial \mathrm{C}_{11} / \partial \mathrm{P}=$ $3.61(\mathrm{GGA}), \partial \mathrm{C}_{12} / \partial \mathrm{P}=4.84(\mathrm{LDA}), \partial \mathrm{C}_{12} / \partial \mathrm{P}=4.74(\mathrm{GGA}) . \mathrm{C}_{44}$ shows a different tendency to $\mathrm{C}_{11}$ and $\mathrm{C}_{12}$, which decreases with the increasing pressure and $\partial \mathrm{C}_{44} / \partial \mathrm{P}=-0.66$ for LDA and $\partial \mathrm{C}_{44} / \partial \mathrm{P}=-1.12$ for GGA.

The calculation results of crystal constants of $\mathrm{B} 4 \mathrm{ZnO}$ are shown in Fig. 6. Both $a$ and $c$ show a decreasing trend with increasing pressure, while the bulk modulus increases with increasing pressure (Fig. 7). The experimental results for crystal constants are $a=3.25 \AA$ and $c=5.21 \AA$ under atmospheric pressure, which lie 

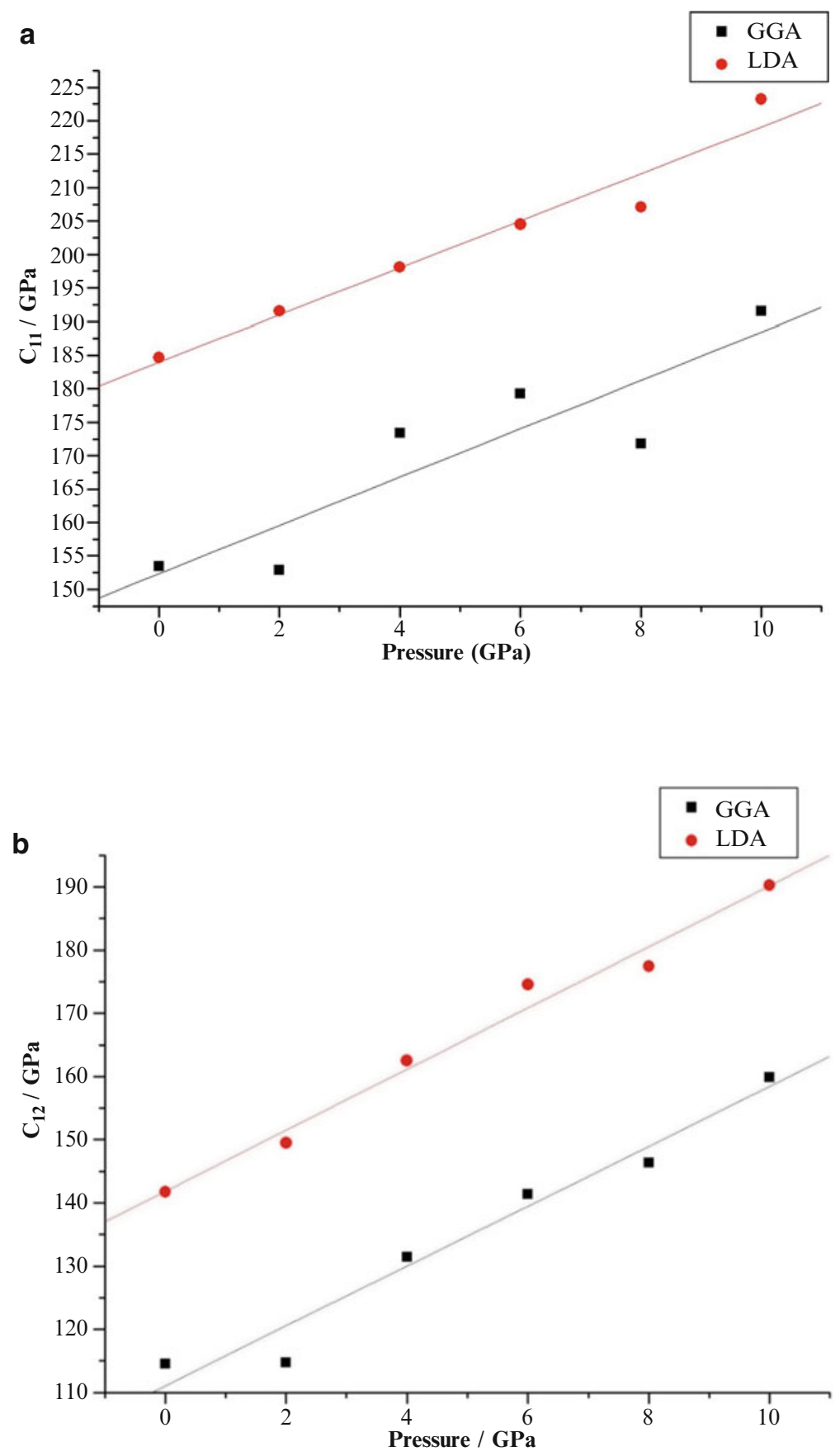

Fig. 5 Relationship between elastic constants and pressure for the $B_{3}$ structure: (a) $C_{11}$, (b) $C_{12}$, (c) $\mathrm{C}_{44}$ 


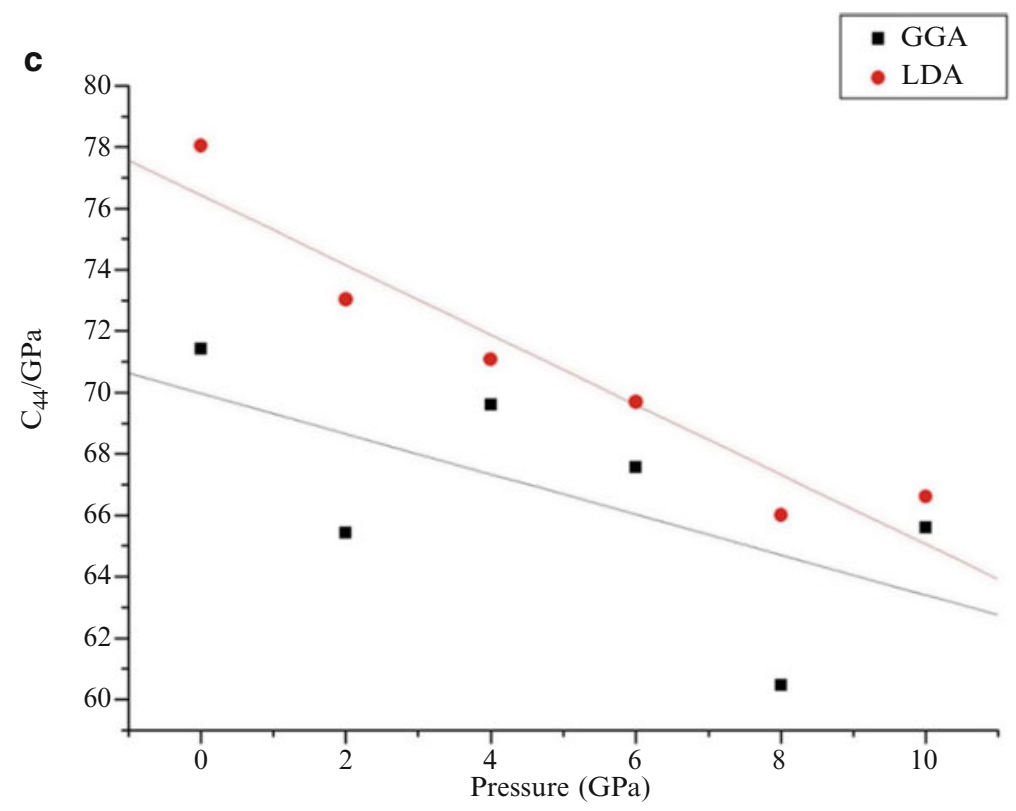

Fig. 5 (continued)

within the range of the GGA $(a=3.285 \AA$ and $c=5.297 \AA)$ and LDA $(a=3.191 \AA$ and $c=5.158 \AA$ ) calculations. As the atoms of a $\mathrm{ZnO}$ crystal become much closer under higher pressure, the repulsion between atoms become much larger, which lead to an increase of the bulk modulus. The results obtained by LDA are larger than the ones by GGA.

$\mathrm{B} 4 \mathrm{ZnO}$ has five independent elastic constants and results are shown in Fig. 8. $\mathrm{C}_{11}, \mathrm{C}_{12}, \mathrm{C}_{13}$ and $\mathrm{C}_{33}$ increase with increasing pressure while only $\mathrm{C}_{44}$ is in the opposite trend. The straight lines shown in Fig. 8 were made according to the least square method. The pressure derivatives are as follows: $\partial \mathrm{C}_{11} / \partial \mathrm{P}=3.35$ (LDA), $\partial \mathrm{C}_{11} / \partial \mathrm{P}=1.93(\mathrm{GGA}), \partial \mathrm{C}_{12} / \partial \mathrm{P}=5.16(\mathrm{LDA}), \partial \mathrm{C}_{12} / \partial \mathrm{P}=2.41(\mathrm{GGA})$, $\partial \mathrm{C}_{13} / \partial \mathrm{P}=4.63(\mathrm{LDA}), \partial \mathrm{C}_{13} / \partial \mathrm{P}=1.75(\mathrm{GGA}), \partial \mathrm{C}_{33} / \partial \mathrm{P}=2.76(\mathrm{LDA})$, $\partial \mathrm{C}_{33} / \partial \mathrm{P}=1.69(\mathrm{GGA}), \partial \mathrm{C}_{44} / \partial \mathrm{P}=-1.06(\mathrm{LDA}), \partial \mathrm{C}_{44} / \partial \mathrm{P}=-0.81(\mathrm{GGA})$.

Table 1 shows the calculated and experimental results for the elastic constants from previous research, together with our results. Most experiments and calculations were carried out under atmospheric pressure; thus only the results with 0 environmental pressure are listed in the table. Compared with other research results, our results are consistent and the errors are small, which gives us confidence in the techniques employed.

The full anisotropic elastic response is not widely used in engineering applications. For simplicity, the material is generally treated as a homogeneous material. Thus only two constants are sufficient to describe the elastic properties without 

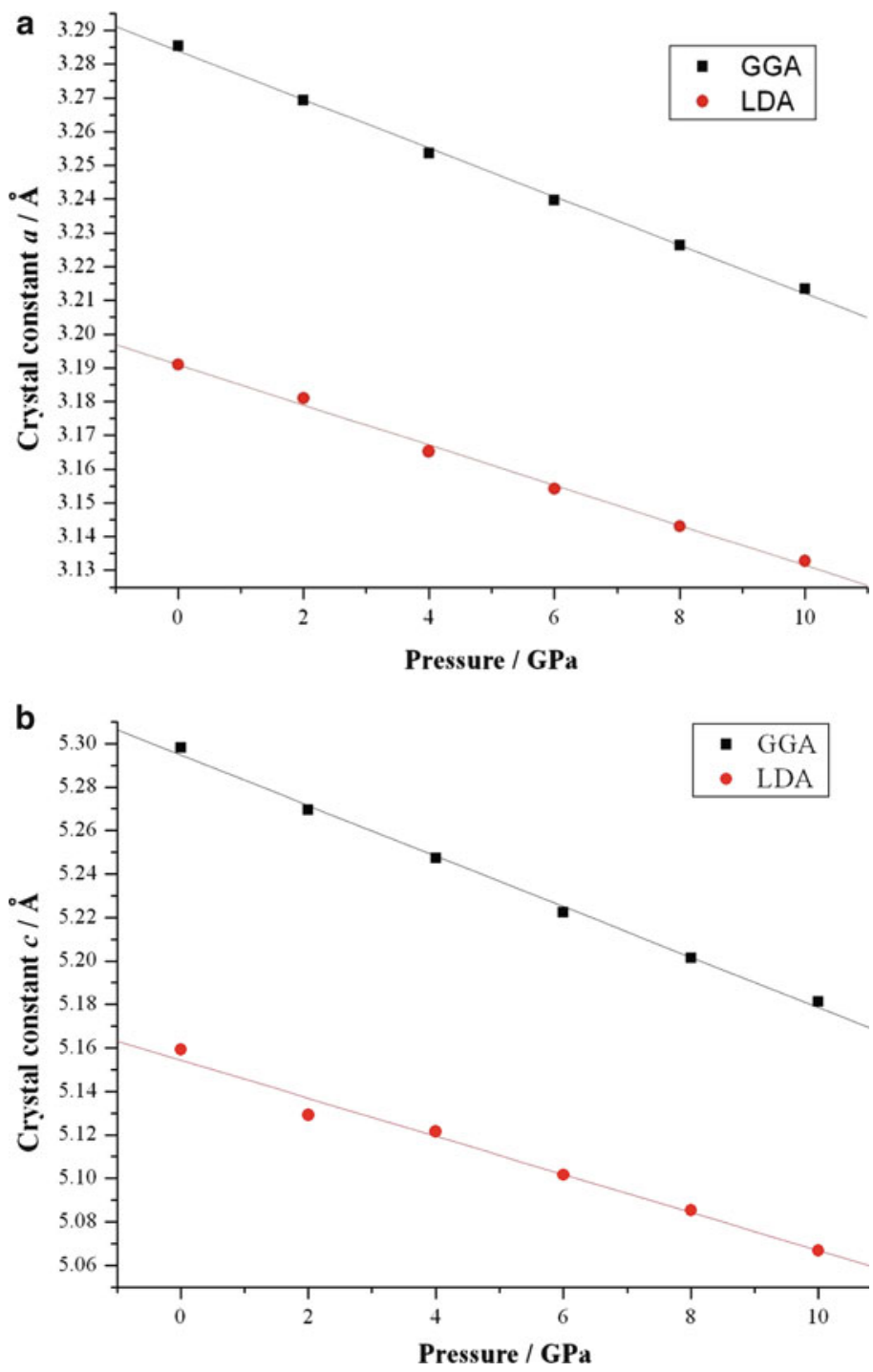

Fig. 6 Relationship between crystal constants and pressure for the $\mathrm{B}_{3}$ structure: (a) $a$, (b) $c$

much error. For zinc blende ZnO, Young's modulus, E, Poisson's ratio v, and the bulk modulus, B, the shear modulus, $\mathrm{G}$, can be determined using the following expressions:

$$
E=\frac{\left(C_{11}+2 C_{12}\right)\left(C_{11}-C_{12}\right)}{C_{11}+C_{12}},
$$




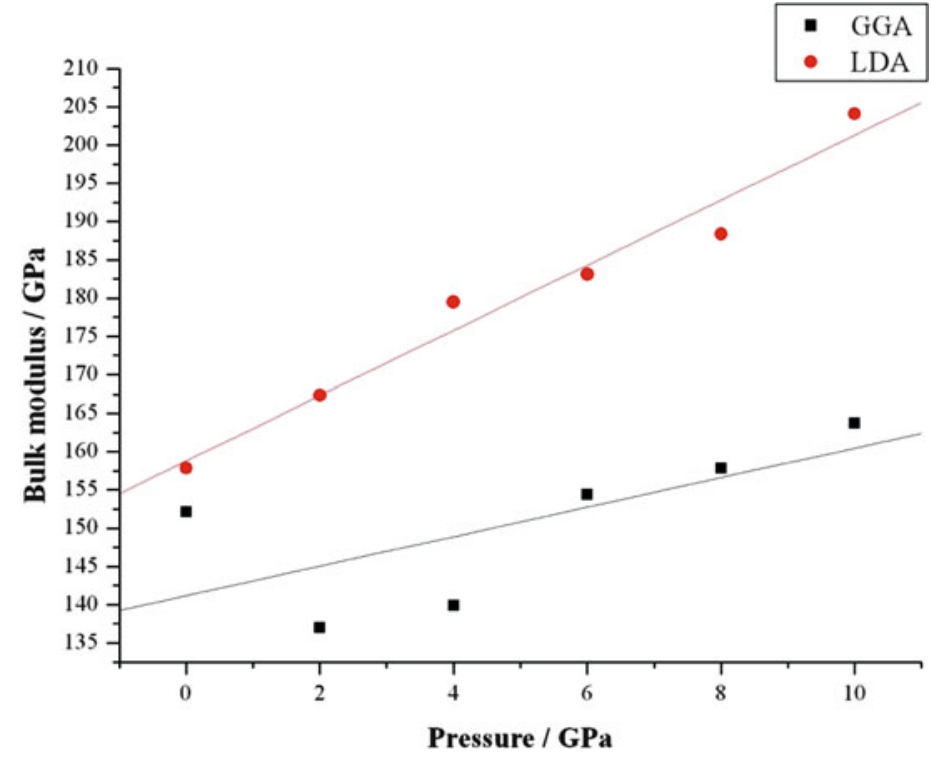

Fig. 7 Relationship between bulk modulus and pressure for the $\mathrm{B}_{4}$ structure

$$
\begin{gathered}
v=\frac{\mathrm{C}_{12}}{\mathrm{C}_{11}+\mathrm{C}_{12}}, \\
\mathrm{~B}=\frac{\mathrm{C}_{11}+2 \mathrm{C}_{12}}{3},
\end{gathered}
$$

and

$$
\mathrm{G}=\frac{\mathrm{C}_{11}-\mathrm{C}_{12}}{2}=\frac{\mathrm{E}}{2(1+v)} .
$$

For $\mathrm{B} 4 \mathrm{ZnO}$, in the isotropic approximation, the bulk modulus, B, shear modulus, G, Young's modulus, E, and Poisson's ratio, v, can be evaluated using the relations [10]:

$$
\begin{gathered}
E=3 \frac{\left(C_{11}+C_{12}-2 C_{13}\right)\left[\left(C_{11}+C_{12}\right) C_{33}-2 C_{13}^{2}\right]}{\left(C_{11}+C_{12}+2 C_{33}-4 C_{13}\right)\left(C_{11}+C_{12}\right)}, \\
v=\frac{C_{13}}{C_{11}+C_{12}}, \\
B=\frac{\left(C_{11}+C_{12}\right) C_{33}-2 C_{13}^{2}}{C_{11}+C_{12}+2 C_{33}-4 C_{13}},
\end{gathered}
$$




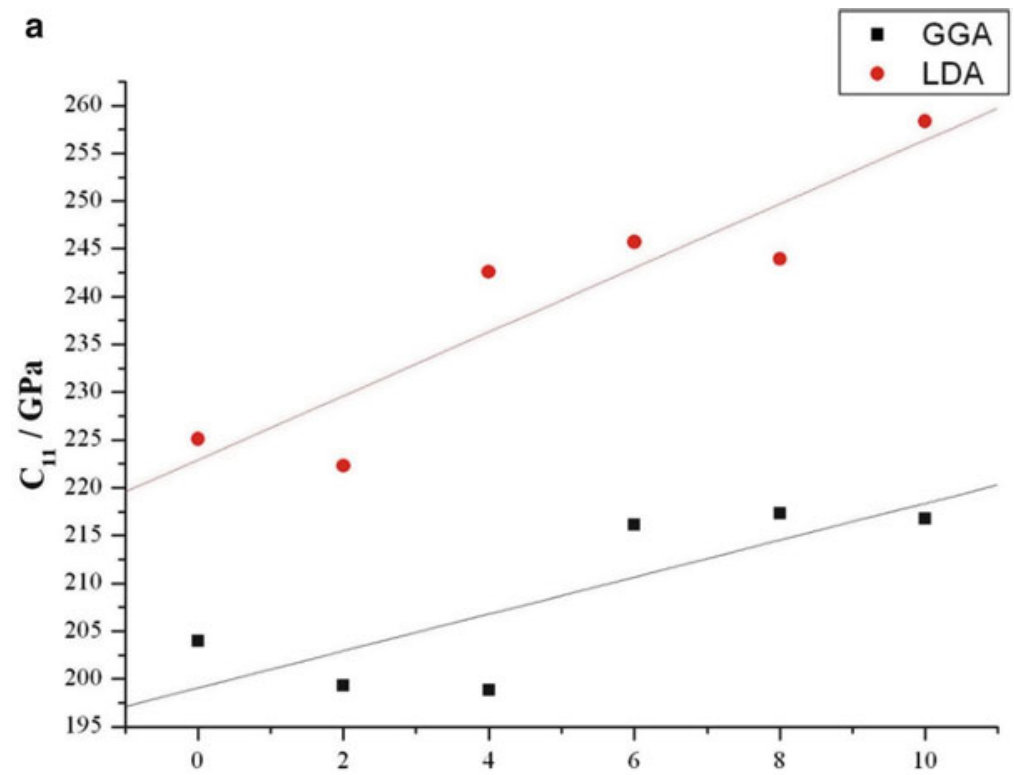

Pressure / GPa

b
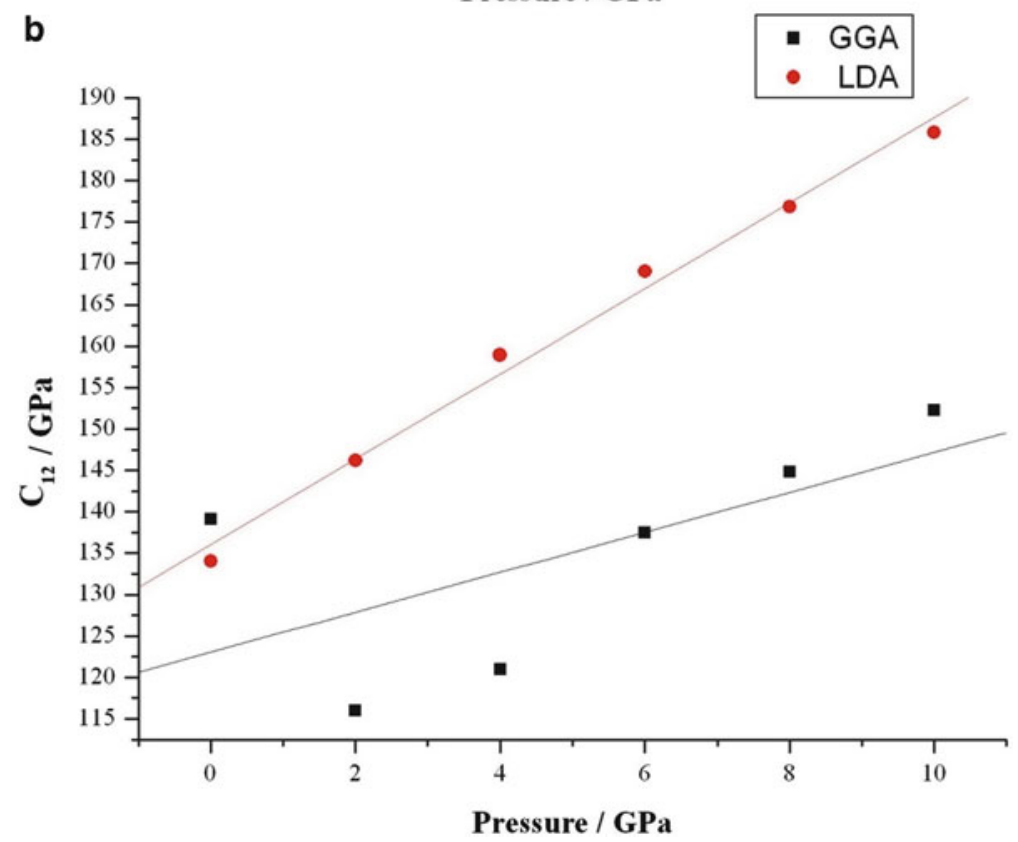

Fig. 8 Relationship between elastic constants and pressure for the $B_{4}$ structure: (a) $C_{11}$, (b) $C_{12}$, (c) $\mathrm{C}_{13}$, (d) $\mathrm{C}_{33},(\mathbf{e}) \mathrm{C}_{44}$ 


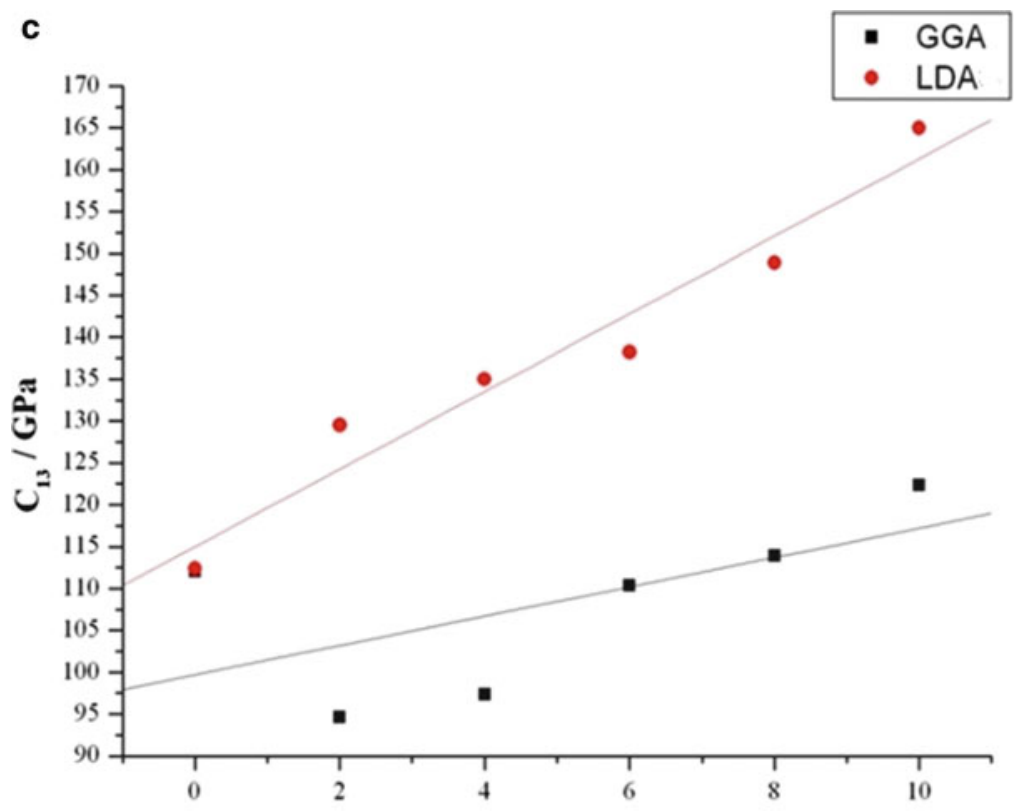

Pressure / GPa

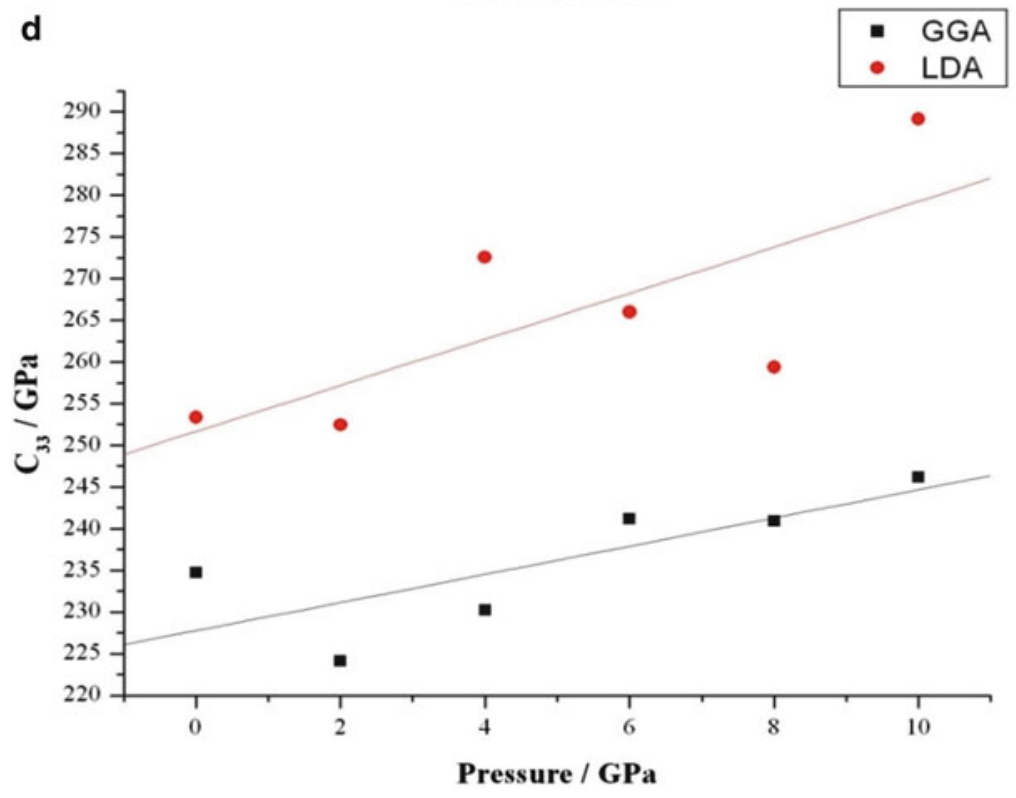

Fig. 8 (continued) 


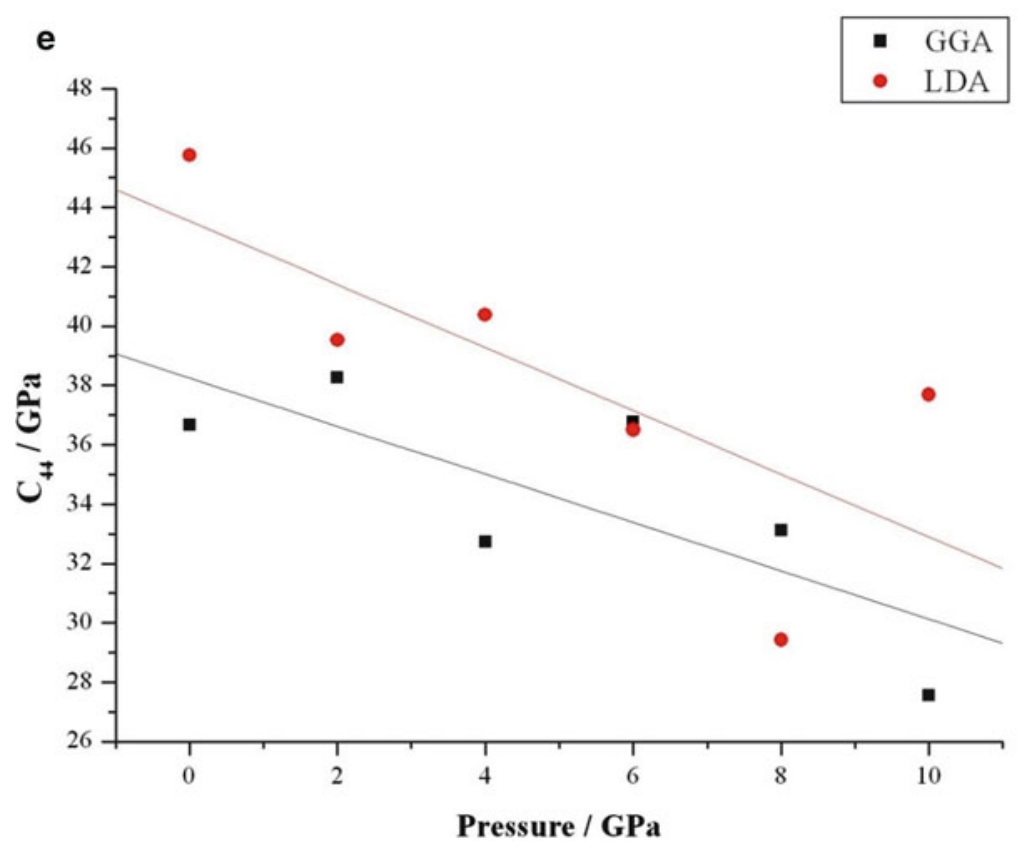

Fig. 8 (continued)

Table 1 Elastic properties of $\mathrm{ZnO}$ with both Zinc blende and Wurtzite structures obtained by experiments and calculations

\begin{tabular}{|c|c|c|c|c|}
\hline$\underline{\text { Parameters }(\mathrm{GPa})}$ & Zinc blend & & Wurtzite & \\
\hline \multirow[t]{2}{*}{$\mathrm{C}_{11}$} & $193[10]$ & 184.7 (LDA) & 209.7 [7], 206 [8], & 223.8 (LDA) \\
\hline & & 153.5 (GGA) & $157[9], 246[10]$ & 204.0 (GGA) \\
\hline \multirow[t]{2}{*}{$\mathrm{C}_{12}$} & $139[10]$ & 141.7 (LDA) & $121.1[7], 117$ [8] & 132.9 (LDA) \\
\hline & & 114.5 (GGA) & 89 [9], $127[10]$ & $139.1(\mathrm{GGA})$ \\
\hline \multirow[t]{2}{*}{$\mathrm{C}_{13}$} & & & $105.1[7], 118$ [8], 83 [9], 105 [10] & 110.9 (LDA) \\
\hline & & & & $112.0(\mathrm{GGA})$ \\
\hline \multirow[t]{2}{*}{$\mathrm{C}_{33}$} & & & 210.9 [7], 211 [8], 208 [9], 246 [10] & 45.8 (LDA) \\
\hline & & & & 234.7 (GGA) \\
\hline \multirow[t]{2}{*}{$\mathrm{C}_{44}$} & $96[10]$ & 78.0 (LDA) & 42.47 [7], 44.3 [8], 38 [9], 56 [10] & 45.7 (LDA) \\
\hline & & $71.4(\mathrm{GGA})$ & & 36.7 (GGA) \\
\hline \multirow[t]{2}{*}{ Bulk modulus } & $154.4[12]$ & 156.0 (LDA) & $142.4[11], 183[12]$ & 156.4 (LDA) \\
\hline & & 127.5 (GGA) & & 152.1 (GGA) \\
\hline
\end{tabular}

and

$$
G=\frac{3\left(C_{11}+C_{12}-2 C_{13}\right)\left[\left(C_{11}+C_{12}\right) C_{33}-2 C_{13}^{2}\right]}{2\left(C_{11}+C_{12}+2 C_{33}-4 C_{13}\right)\left(C_{11}+C_{12}+C_{13}\right)}=\frac{E}{2(1+v)}
$$




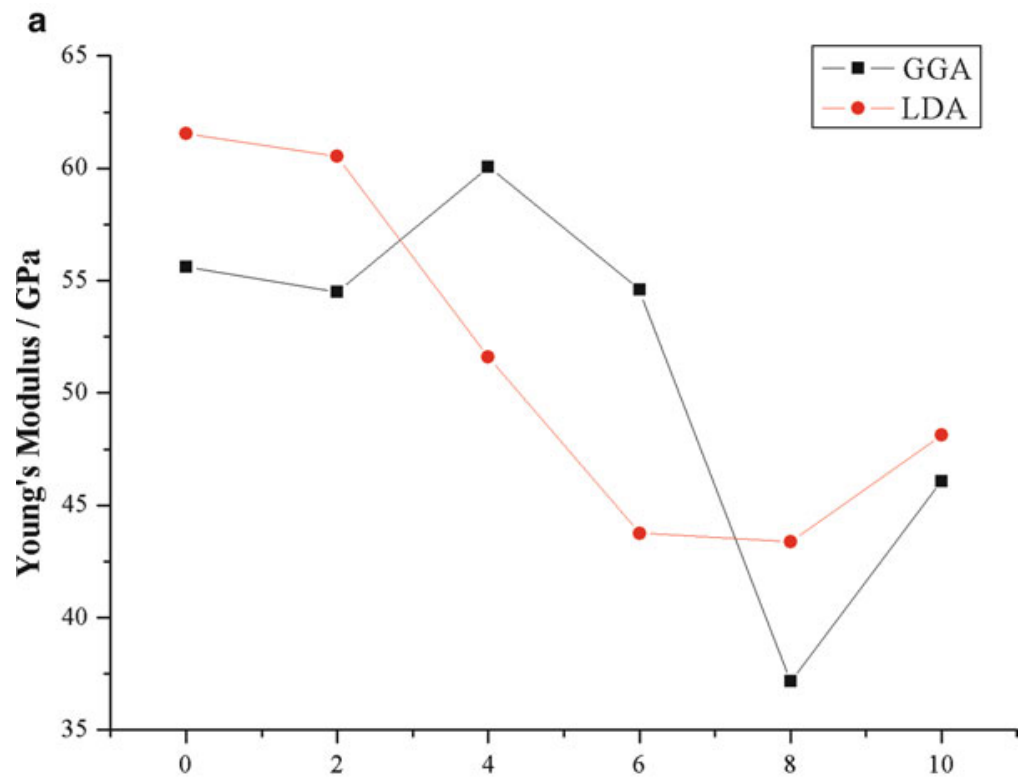

Pressure / GPa

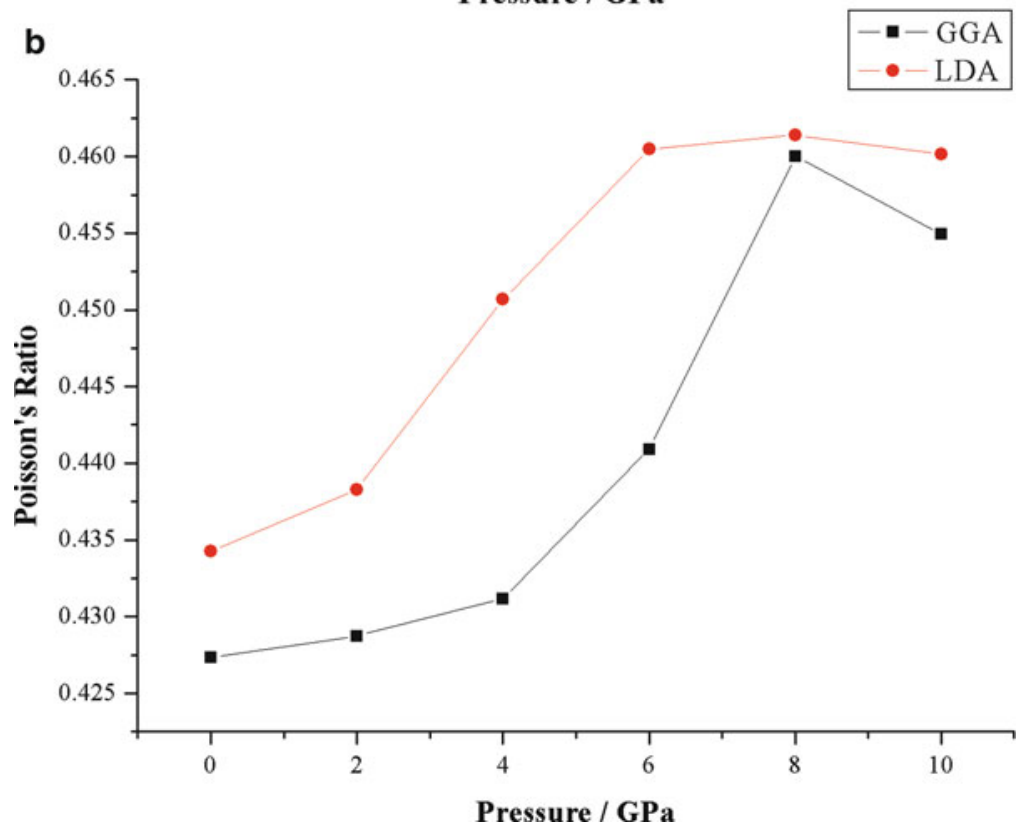

Fig. 9 Relationship between young's modulus, poisson's ratio and pressure for the $\mathrm{B}_{3}$ structure: (a) Young's modulus, (b) Poisson's ratio 


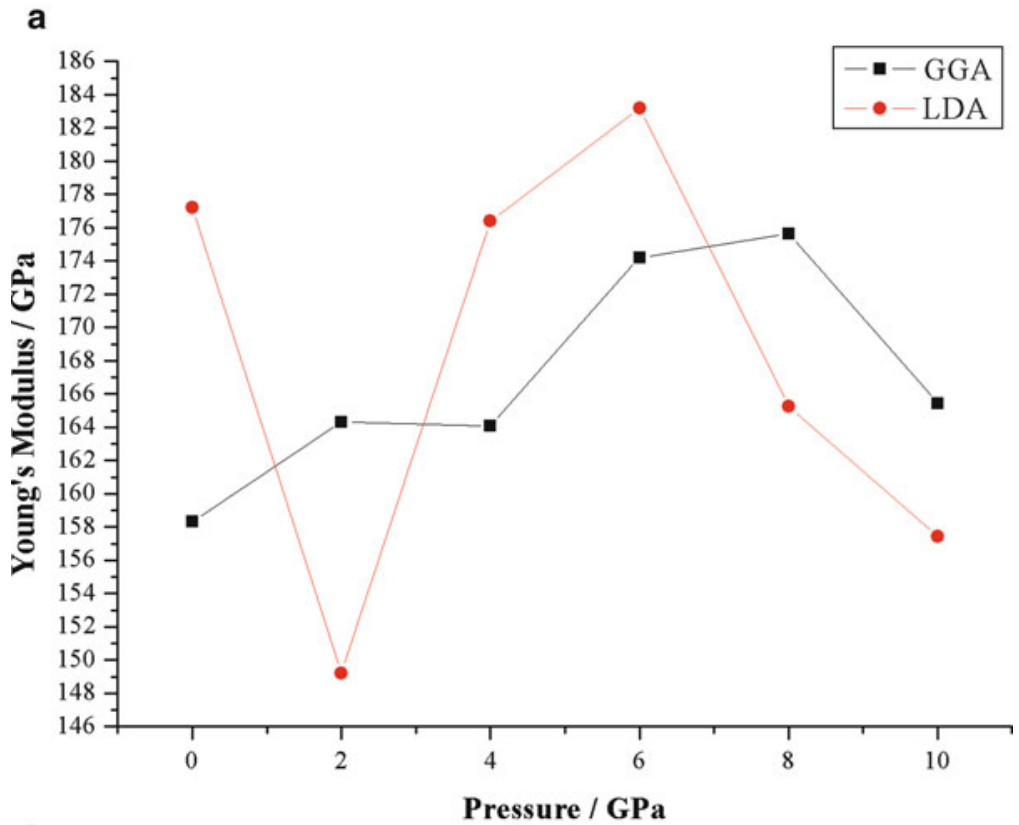

b

Pressure / GPa

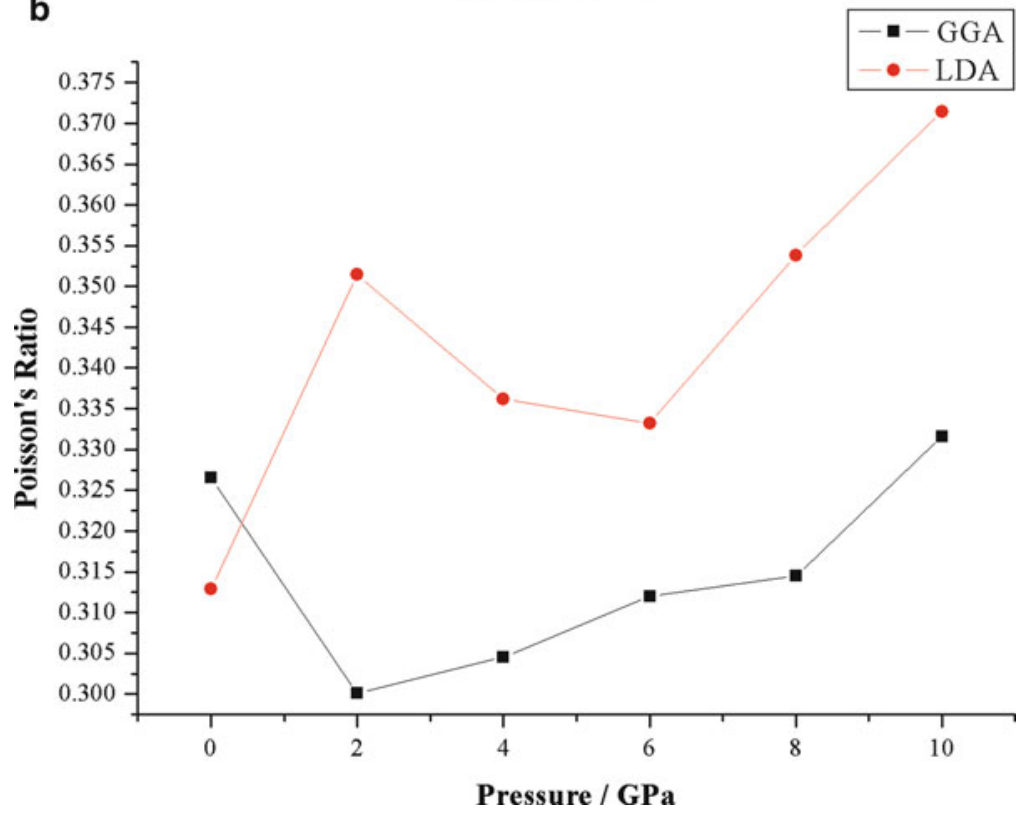

Fig. 10 Relationship between young's modulus, poisson's ratio and pressure for the $\mathrm{B}_{4}$ structure: (a) Young's modulus, (b) Poisson's ratio 
For $\mathrm{B} 3 \mathrm{ZnO}$ under atmospheric pressure, the Young's moduli are 61.7 $\mathrm{GPa}$ (LDA) and 55.7 GPa (GGA), while Poisson's ratio is 0.43 for both models. For B4 $\mathrm{ZnO}$ under the same conditions, Young's modulus is $180 \mathrm{GPa}$ (LDA) and $158 \mathrm{GPa}$ (GGA), and Poisson's ratio is 0.31 (LDA) and 0.33 (GGA). The relationships between Young's modulus, Poisson's ratio and pressure for B3 structures are shown in Fig. 9 while Fig. 10 shows the results for B4. From the figures, we find that the Young's moduli for the two structures do not simply increase with increasing pressure. The Young's modulus for B3 is lower at higher pressure, while the result for B4 was irregular. Poisson's ratios for the two structures become larger at higher pressure, which reflects the observation that the compressibility decreases with increasing environmental pressure. The shear modulus G, which is not an independent constant, can be directly deduced from Young's modulus and Poisson's ratio.

\section{Conclusions}

In this paper, calculations based on DFT were carried out to investigate the elastic properties and crystal structures of $\mathrm{ZnO}$ single crystals under high pressure. Both zinc blende and wurtzite structures were included in the calculation. CASTEP with both GGA and LDA was employed to investigate the behavior of longitudinal and shear modes for a $\mathrm{ZnO}$ single crystal under high pressure. The obtained results accord well with previous works. For zinc blende $\mathrm{ZnO}$, results obtained by DFT show that the bulk modulus, $\mathrm{C}_{11}$ and $\mathrm{C}_{12}$ increase with increasing pressure, while the crystal lattice constant and $\mathrm{C}_{44}$ show a different trend. The elastic constants obtained by LDA are larger than GGA, and the experimental lattice constant lies between the results of the two approximations. Further investigations on Young's moduli showed that E decreases with increasing pressure for B3 and no rules were found for B4. Under still higher pressure, Poisson's ratio was found to be larger. These results are helpful to widen the application of $\mathrm{ZnO}$ nanomaterials.

Acknowledgements This work was jointly supported by the National Natural Science Foundation of China (NSFC, Grant Nos. 60936001, 11072244, 11011120245 and 11021262) and the National Basic Research Program of China (973 Program, Grant No. 2007CB310500).

\section{References}

1. Black, K., Chalker, P.R., Jones, A.C., King, P.J., Roberts, J.L., Heys, P.N.: A new method for the growth of zinc oxide nanowires by MOCVD using oxygen-donor adducts of dimethylzinc. Chem. Vapor Depos. 16, 106-111 (2010)

2. He, F.Q., Zhao, Y.P.: Growth and optical properties of peculiar ZnO tetrapods. J. Phys. D 40 , 1211-1211 (2007) 
3. Li, W.J., Shi, E.W., Zhong, W.Z., Yin, Z.W.: Growth mechanism and growth habit of oxide crystals. J. Cryst. Growth 203, 186-196 (1999)

4. Okada, T., Kawashima, K., Ueda, M.: Ultraviolet lasing and field emission characteristics of ZnO nano-particle-assisted pulsed-laser ablation deposition. Appl. Phys. A 81, 907-910 (2005)

5. Wang, B.B., Xie, J.J., Yuan, Q.Z., Zhao, Y.P.: Growth mechanism and joint structure of ZnO tetrapods. J. Phys. D: Appl. Phys. 41, 102005 (2008)

6. Music, S., Saric, A., Popovic, S.: Formation of nanosize $\mathrm{ZnO}$ particles by thermal decomposition of zinc acetylacetonate monohydrate. Ceram. Int. 36, 1117-1123 (2010)

7. Bateman, T.B.: Elastic moduli of single-crystal zinc oxide. J. Appl. Phys. 33, 3309 (1962)

8. Heo, Y.W., Tien, L.C., Kwon, Y., Norton, D.P., Pearton, S.J., Kang, B.S., Ren, F.: Depletionmode $\mathrm{ZnO}$ nanowire field-effect transistor. Appl. Phys. Lett. 85, 2274-2276 (2004)

9. Carlotti, G., Socino, G., Petri, A., Verona, E.: Acoustic investigation of the elastic properties of ZnO films. Appl. Phys. Lett. 51, 1889-1891 (1987)

10. Catti, M., Noel, Y., Dovesi, R.: Full piezoelectric tensors of wurtzite and zinc blende $\mathrm{ZnO}$ and ZnS by first-principles calculations. J. Phys. Chem. Solids 64, 2183-2190 (2003)

11. Desgreniers, S.: High-density phases of $\mathrm{ZnO}$ : structural and compressive parameters. Phys. Rev. B 58, 14102-14105 (1998)

12. Jaffe, J.E., Hess, A.C.: Hartree-Fock study of phase-changes in $\mathrm{ZnO}$ at high pressure. Phys. Rev. B 48, 7903-7909 (1993)

13. Zang, J.L., Yuan, Q.Z., Wang, F.C., Zhao, Y.P.: A comparative study of young's modulus of single-walled carbon nanotube by CPMD, MD and first principle simulations. Comput. Mater. Sci. 46, 621-625 (2009)

14. Yuan, Q.Z., Zhao, Y.P.: Precursor film in dynamic wetting, electrowetting and electro-elastocapillarity. Phys. Rev. Lett. 104, 246101 (2010)

15. Yuan, Q.Z., Zhao, Y.P.: Hydroelectric voltage generation based on water-filled single-walled carbon nanotubes. J. Am. Chem. Soc. 131, 6374-6376 (2009)

16. Ceperley, D.M., Alder, B.J.: Ground state of the electron gas by a stochastic method. Phys. Rev. Lett. 45, 566-569 (1980)

17. Perdew, J.P., Zunger, A.: Self-interaction correction to density-functional approximations for many-electron systems. Phys. Rev. B 23, 5048-5079 (1981)

18. Perdew, J.P., Wang, Y.: Accurate and simple analytic representation of the electron-gas correlation energy. Phys. Rev. B 45, 13244-13249 (1992) 